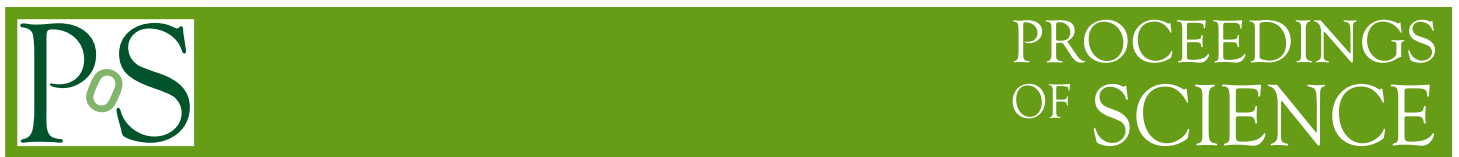

\title{
Dissipation and vacuum selection
}

\author{
Ana Achúcarro ${ }^{1}$, Nuno D. Antunes ${ }^{2}$, Filipe Freire ${ }^{1 *} \&$ Petja Salmi $i^{1,2}$ \\ ${ }^{1}$ Inst. Lorentz, Univ. Leiden, P. O. Box 9506, 2300 RA Leiden, The Netherlands \\ ${ }^{2}$ Centre for Theoretical Physics, University of Sussex, Falmer, Brighton BN1 9QJ, U. K. \\ E-mail: freiredlorentz.leidenuniv.nl
}

\begin{abstract}
We study the symmetry breaking pattern of a model with an $O(4)$ scalar field sector, with both charged and neutral fields, interacting with a photon bath. Here it is shown that a selective condensation of the neutral fields, that are not directly coupled to photons, can be achieved in the presence of a minimal "external" dissipation, i.e. not related to interactions with a bath. Preliminary results from simulations illustrating defect formation are also discussed. This should be relevant in the early universe or in heavy-ion collisions where dissipation occurs due to expansion.
\end{abstract}

29th Johns Hopkins Workshop on current problems in particle theory: strong matter in the heavens 1-3 August

Budapest

* Speaker. 


\section{Introduction}

In this talk I will report on an ongoing investigation about the role of dissipation in biasing vacuum selection after a symmetry breaking phase transition. This work was motivated by a mechanism suggested by Nagasawa and Brandenberger [1] to stabilise non-topological classical solutions by out-of-equilibrium effects. These authors studied a model with $O(4)$ scalar field sector with charged and neutral scalars in contact with a thermalised photon bath. Assuming that only the char ged fields receive thermal corrections they argued that the non-topological strings were temporarily stabilised.

The analysis in [1] is focused on the stability of embedded strings when immersed in a thermalised plasma. However, the primordial question of whether the formation of the defect is dynamically favoured has never been addressed. By looking at the requirements that favour their formation we found a close link between vacuum selection and an "external" dissipation, i.e. a source of dissipation that is not related to the interactions between the system and the heat bath. For example, in a cosmological context this type of dissipation is naturally associated with the expansion of the universe. As we will show as result of this the decoupled fields stabilise in steady states that can be characterised by an effective temperature $T_{\text {eff }}<T$. Our initial results have by published in [2].

The vacuum selection we discuss can take place in the early universe and in heavy-ion collisions. In the early universe, the most relevant areas for applications are in the studies of preheating at the end of inflation [3] and defect formation in non-equilibrium cosmological phase transitions [4].

The mechanism we investigate will be illustrated in an $O(4)$ scalar field theory in $3+1$ dimensions as in [1]. Some general assumptions are required to specify the properties of the model. This are discussed in detail in [2]. Let us summarised them here. In the context of our work the field theory is an effective model for the soft long-wavelength modes of a system coupled to a heat bath. At low temperatures the system has a symmetry broken phase and the symmetry is restored above a critical temperature $T_{c}$. In the ordered phase, two of the scalar fields, say for definiteness $\phi_{1,2}$, have decoupled from the heat bath, while the remaining fields, say $\phi_{3,4}$, stay coupled. This situation is natural if for example if the fields $\phi_{1,2}$ are neutral and $\phi_{3,4}$ are charged and the heat bath consists of photon. Furthermore, the coupling between $\phi_{3,4}$ and the bath is assumed to be much stronger than the scalar self-coupling. By the time $\phi_{3,4}$ thermalise, the $\phi_{1,2}$ fields have not yet reached a steady state.

The most interesting effect occurs when the "external" dissipation is much smaller than the dissipation in the coupled fields due to their interaction with the bath. We have that at vanishing scalar self-coupling the limit $\eta_{1,2} \rightarrow 0$, i.e. the "external" sources of dissipation are "switched off", is singular [2]. This changes the symmetry breaking pattern even for small values of $\eta_{1,2}$. We emphasise that the origin of the vacuum manifold reduction in our analysis occurs without having to call for out-of-equilibrium effects as in [1]. The effect follows from the existence of different steady states for the various fields due to the "external" source of dissipation. The vacuum selection takes place above a small critical dissipation which occurs when the neutral fields stabilise at a "cold" enough $T_{\text {eff. }}$.

Our simulations are governed by phenomenological Langevin equations describing the dy- 
namical evolution of the fields. These equations have been previously used in a relativistic context to study non-equilibrium phenomena in cosmological phase transition $[5,6,7]$. There are known limitations to the use of these equations, nevertheless they provide an economic and qualitative good description of the different processes involved in the dynamic evolution of the fields where the coupling to the heat bath is expressed by rapidly fluctuating fields and the dissipation effects are expressed by viscosity terms. In particular, this makes it easy to analyse the effects of dissipation terms that are not related to interactions with the heat bath.

\section{Decoupling and dissipation}

We analyse the field dynamics in a system that undergoes a symmetry breaking transition and where different fields sectors in the system reach distinct "thermalised" states with some heat bath. The nature of the bath will be characterised below. Some fields arrive at a standard thermalised state at the temperature of the bath after a relatively short relaxation time. The remaining fields stay out of equilibrium for a longer period, which can still be small compared to observation times. The fields that take longer to either thermalise or reach a distinct steady state are weakly or indirectly coupled to a heat bath. For convenience we refer to them as decoupled. In particular, we are interested in the situation where the decoupled fields condense following a finite temperature phase transition. For this to happen these fields must lose most of their energy and for this reason we follow closely the role of dissipation.

In order to discuss a setting where this scenario can be realised we use a model with an $O(4)$ scalar sector with a Lagrangean,

$$
\mathscr{L}=\frac{1}{2} \partial_{\mu} \phi_{i} \partial^{\mu} \phi_{i}-\frac{\lambda}{4}\left(\phi_{i} \phi_{i}-v^{2}\right)^{2}, i=1,2,3,4,
$$

to describe the propagation and self-interactions of soft modes. We consider $\phi_{1}$ and $\phi_{2}$ to be neutral scalars and $\phi_{3}$ and $\phi_{4}$ to be the constituents of a charged scalar $\phi^{ \pm}=\left(\phi_{3} \pm i \phi_{4}\right) / \sqrt{2}$ with respect to a $U(1)$ charge. For concreteness let it be $U(1)_{\mathrm{EM}}$. Therefore, $\phi^{ \pm}$is coupled to a bath of photons while the neutral scalars are not. In a more comprehensive analysis the effects of the fluctuations from the hard modes of the scalar fields are also to be taken into account [2].

With this simple model we can aim at understanding better how a small dissipation can play a role in selecting the vacuum. This is the effect we emphasise here. Alongside, we lay the conditions for vacuum selection that favour the formation of stable embedded structures.

As mentioned, we assume that the coupling between the charged scalars and the photon bath is much stronger than the scalar self-coupling. Without this assumption leading to an effective separation of scales no non-trivial selection seems to take place. With these conditions, we expect the charged scalars to thermalise "quickly". Their relaxation time sets the scale for what we will refer as a quick thermalisation time. In practice, at the observation scale, the charged scalars can be said to remain in equilibrium. The neutral scalars have of course a longer thermalisation time and are never far away from thermal equilibrium.

One of our aims is to express quantitatively the distinction between the steady state reached by the neutral and the charged fields. If the decoupled fields have no direct process to dissipate their energy they reach a thermal equilibrium state at the temperature $T$ of the photon bath. This 
thermalisation occurs because the decoupled fields are not completely cut off from the photon bath due to the quartic scalar self-interaction. The rate at which the neutral fields thermalise depends on the strength of $\lambda$. Elsewise, if they dissipate due to the expansion of the system as it cools, as in the early universe or heavy-ion collisions, the steady state they reach is "colder". This effect leads to their selective condensation and suggests the use of an effective temperature $T_{\text {eff }}$ as a way to parametrise the distinct steady states.

\section{Vacuum biasing: simulations}

In order to simulate the dynamics of the fields we use phenomenological Langevin equations

$$
\left[\left(\partial_{t}^{2}-\nabla^{2}\right)-\mu^{2}+\lambda \sum_{k=1}^{4} \phi_{k}^{2}+\eta_{i} \partial_{t}\right] \phi_{i}=\xi_{i}
$$

with $\mu^{2}=\lambda v^{2}, v$ the $T=0$ vacuum expectation value (VEV), and $\eta_{i}$ and $\xi_{i}$ are respectively the viscosity coefficients and the Gaussian noises. For the fields that couple to the photon bath and thermalise at its temperature $T$, we have

$$
\left\langle\xi_{i}(\vec{x}, t) \xi_{j}\left(\vec{x}^{\prime}, t^{\prime}\right)\right\rangle=\Omega_{i} \delta_{i j} \delta^{(3)}\left(\vec{x}-\vec{x}^{\prime}\right) \delta\left(t-t^{\prime}\right) .
$$

where $\beta \Omega_{i}=2 \eta_{i}$, with $\beta=1 / T$, according to the fluctuation-dissipation theorem. Below $T_{c}$ this relation applies only for $i=3,4$, while in the disordered phase it applies to all fields. In the ordered phase the neutral fields are decoupled from the bath, $\xi_{i}=0$, but $\eta_{i} \neq 0$. The non vanishing value of these coefficients are due to an "external" source of dissipation.

We use a discretised version of (3.1) in three dimensional square lattices with $50^{3}$ to simulate the evolution of the $O(4)$ model (2.1). A leap-frog algorithm with time step $\delta_{t}=0.05$ is used. Larger lattices of $100^{3}$ have been used to verify the stability of our results. A Gaussian random number generator is used for the rapidly changing fluctuations. All quantities are measured in units of the VEV $v$. The dimensionless quantities are identified with a tilde, e.g. $\widetilde{v}=1$ and $\widetilde{\beta}=v / T$. When choosing the lattice spacing we need to ensure that the modes with wavelength longer than $\sim T^{-1}$ are not cut off. In our runnings, merely for reference, we took physical scales from the chiral symmetry breaking effective mean field model. By using a lattice spacing $\widetilde{\Delta x}=0.25$ for $v=93$ $\mathrm{MeV}$ we can work up to temperatures of approximately $T \sim 350 \mathrm{MeV}$.

We run our simulations for successive temperatures of the heat bath determined by $\Omega_{+}=$ $2 \eta_{+} T$, the amplitude for the noise of the charged scalars. Starting from a temperature $T>T_{c}$ we bring down the temperature across $T_{c}$. While in the disordered phase all the fields are taken to be in contact with the heat bath. It is only for $T<T_{c}$, when the VEV starts to increase, that the neutral fields are decoupled.

In Figure 1, we plot the order parameters for the condensates of neutral and charged scalars against the inverse temperature $\beta$. These are, respectively, $\left\langle\left|\phi_{1} \phi_{2}\right|\right\rangle$ and $\left\langle\left|\phi_{3} \phi_{4}\right|\right\rangle$, where $\left\langle\left|\phi_{i} \phi_{j}\right|\right\rangle=$ $\sqrt{\sum_{n=i, j}\left(\frac{1}{V} \int_{V} \phi_{n}(x)\right)^{2}}$ are averages over the entire lattice. As the details of the decoupling of the neutral fields are not known we show the curves for three types of decoupling. For the runnings in Figure 1, we observe a bias for the condensation of the neutral fields. We used $\eta_{0} / \eta_{+}=1$ to emphasise the case when the condensation of the neutral sector is strongly favoured. 


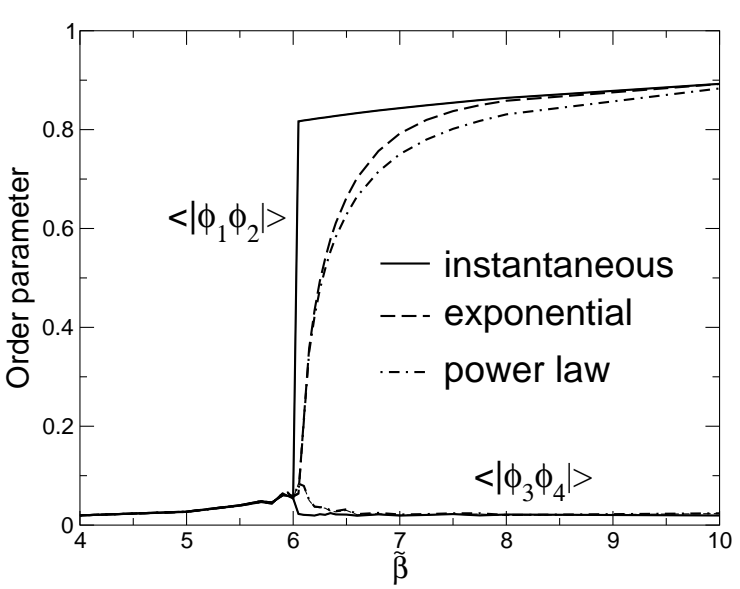

Figure 1

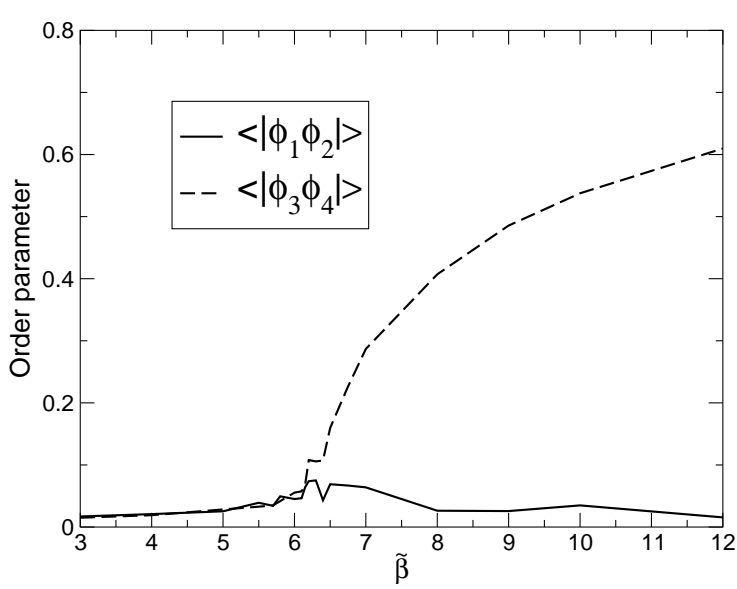

Figure 2

For the large value of $\eta_{0}$ used for Figure 1 all the scalars have an equally effective channel to cool as $T$ decreases. However, the fluctuations from the interaction with the heat bath slow down the dissipation of the charged fields. This favours the neutral fields to roll down more effectively to the bottom of the potential and condense.

The neutral fields are not blind to the photon bath due to the scalar self-coupling. They dissipate through the viscosity term while gaining energy via the scalar self-coupling. But, as long as the effects of fluctuations are small and energy is effectively dissipated, which occurs when $\lambda$ is not too large and $\eta_{0}$ not too small compared to $\eta_{+}$, the neutral scalars continue to monopolise the vacuum. This situation changes when $\eta_{0}$ becomes too small.

In Figure 2, where we set $\eta_{0}=0$ the charged scalars condense. This suggests that as $\eta_{0}$ is decreased below some critical value the charged fields condense instead of the neutral ones. This results in a "superconducting" background where the photons are massive. However, this situation violates our working assumption that the photon bath is thermalised. The condensation of the charged scalars in Figure 2 is an artifact of our simulations.

We predict the existence of a critical dissipation $\eta_{\mathrm{cr}}=\eta_{0} / \eta_{+}$in the interval $0<\eta_{\mathrm{cr}}<1$. A precise determination of $\eta_{\mathrm{cr}}$ at this phenomenological stage of our study does not justify the dedicated effort it requires. At most this critical value indicates the end of the validity of our working conditions and a qualitative change in the nature of the condensation. This matter is further discussed in $[2]$.

Let us now analyse the interplay between the various parameters in the model. We look at the kinetic energy of each set of fields to learn how thermalisation is affected by the ratio of the viscosity coefficients $\eta=\eta_{0} / \eta_{+}$and the scalar coupling $\lambda$. In Figure 3 the time evolution of the kinetic energies for both types of scalar fields are plotted. The parameters $\widetilde{\eta}_{0}=0.005, \widetilde{\eta}_{+}=1$ and $\widetilde{\beta}=6.5$, all in units of the VEV $v$ in a $100^{3}$ box, are the same for all the curves, whereas three different values for $\lambda$ are used. The equilibrium curve corresponds to the coupled fields that thermalise quickly.

The most interesting feature of Figure 3 is the $\lambda$ dependence of the asymptotic values for the kinetic energy of the decoupled fields. We can interpret these as effective equilibration temperatures $T_{\text {eff }}$. We observe that the larger $\lambda$ is, the faster the decoupled fields approach a steady state. 


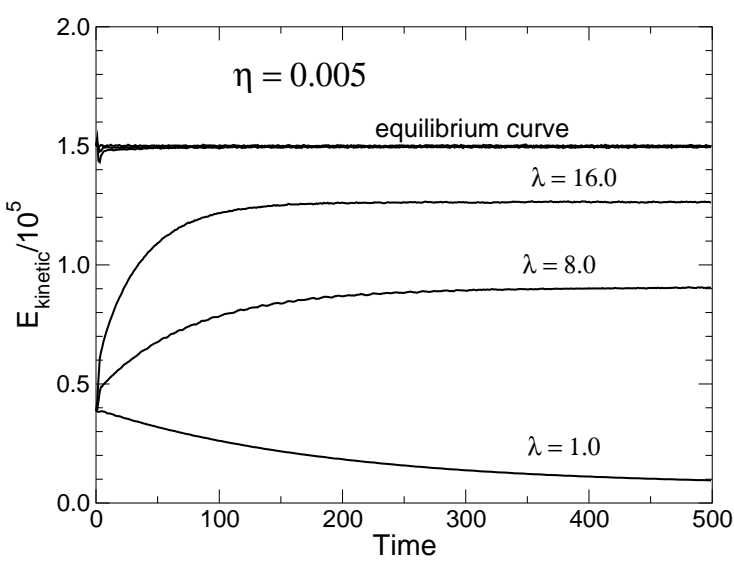

Figure 3

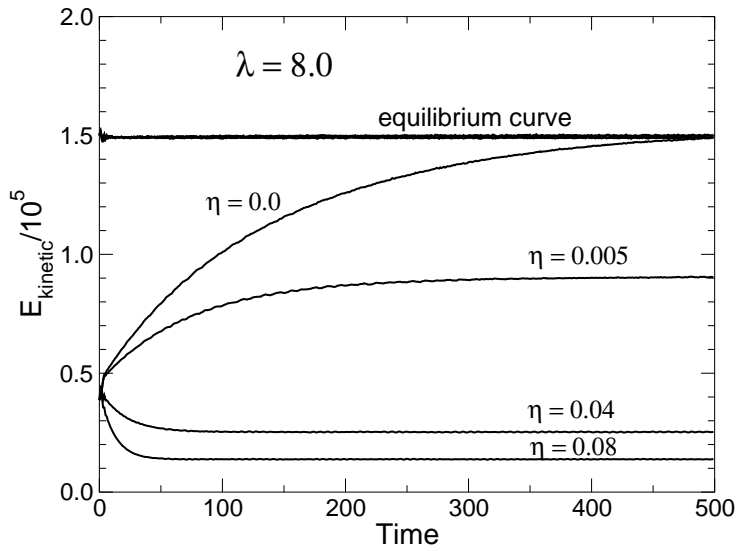

Figure 4

This is to be expected as the decoupled fields interact indirectly with the photon bath via the quartic scalar coupling. It explains not only the shorter relaxation times for larger values of $\lambda$ but also the higher $T_{\text {eff }}$ values which get closer to the temperature of the bath. The value of the asymptotic kinetic energies is independent of the initial conditions.

In Figure 4 we complement the curves shown in Figure 3 by keeping now the same scalar coupling for all the curves, here we use $\lambda=8$, and vary $\eta_{0}$, or equivalently $\eta$ as we again set $\eta_{+}=1$. We observe that the larger the viscosity coefficient $\eta_{0}$ the faster the decoupled fields equilibrate. On the other hand, as $\eta$ increases $T_{\text {eff }}$ shifts away from $T$. The asymptotic steady state approaches the equilibrium curve only in the opposite limit, i.e. when the external dissipative channel is "switch off". In this case, the kinetic energy does eventually reach the value expected by equipartition but at a very slow rate set by the magnitude of $\lambda$ via the fluctuations mediated by the scalar coupling.

If in addition to what we have discussed here we introduce fluctuation terms to the evolution equations for the decoupled fields, the external dissipation can not be neglegible with relation to both thermal dissipation coefficients [2] in order to have observable effects. In our simulations the effects are noticeable because $\eta_{+} \gg \eta_{0}^{\text {ext }}=\eta_{0}$, but $\eta_{0}^{\text {ext }}>\eta_{0}^{\mathrm{fl}}=0$. The first of these two inequalities justifies neglecting non thermal terms in the equations for the coupled scalars. In general, the biasing should occur as long as $\eta_{0}^{\text {ext }} \gtrsim \eta_{0}^{\mathrm{fl}} \neq 0$. Furthermore, adding an explicit coupling to a heat bath would only have the effect of decreasing the relaxation time.

\section{Summary and outlook}

We have shown that under quite general conditions a non thermal dissipation in the evolution of the neutral fields effectively reduces the vacuum manifold of a system described by an $O(4)$ scalar model from $S^{3}$ to $S^{1}$, above a small dissipation threshold. From this analysis we identify the conditions that favour the formation and stabilisation of embedded defects as first argued by Nagasawa and Brandenberger [1].

The vacuum manifold reduction is due to the existence of different asymptotic steady states for the two types of scalar fields considered. This effect is caused by an "external" source of dissipation, in the sense that it does not arise from fluctuations resulting from interactions with the 
photon bath. The neutral field steady state is characterised by an effective temperature $T_{\text {eff }}<T$, "colder" than the photon bath, which drives their selective condensation.

The remarkable feature is that even a small amount of "external" dissipation can be sufficient to cause qualitatively distinct effects, such as the vacuum selection. In principle, one would be inclined to neglect the possible effects of a small amount of dissipation. For instance, the asymptotic state of the charged field sector is hardly affected by the "external" dissipation. What changes things is the existence of an indirect thermal dissipation due to the coupling between the coupled and decoupled fiels. As a result, the external dissipation needs at least to be of the order of the indirect one which is in principle smaller than the direct thermal dissipation.

We can naturally generalise the system to a more realistic one where the external dissipation is considered in both scalar field sectors. However, for the charged field sector this leads only to negligible corrections. We can also consider that neutral scalars are coupled directly to the heat bath although with a much weaker coupling than the charged scalars so that the resulting thermal dissipation for the former does not dominate over the non thermal external dissipation. Under these general conditions our results on vacuum selection are not qualitatively changed.

Corrections to the scalar fields potential coming from the interactions with the gauge bosons can also be taken into account. However, they should not play an important role for our analysis. We know from the work of Nagasawa and Brandenberger [1] that the asymmetry created by the decoupling from the neutral fields from the photon bath bias the effective potential in a way that stabilises non topological defects when immersed in a photon plasma. Moreover, equilibrium thermal corrections to the potential tend to reduce the instability of these embedded configurations [8]. Therefore, at a perturbative level we do not expect corrections to counteract the vacuum selection we analyse here. A less investigated difficulty, but potentially an important one, is the contribution from very soft photons. Because of infra-red divergences reliable corrections similar to those in $[9,10]$ are not to our knowledge currently available. More dedicated simulations including the full dynamics of both the scalars and the gauge bosons are necessary to clarify this problem.

Finally, we discuss preliminary results from simulations illustrating the formation of defects resulting from the vacuum selection mechanism presented here. We have looked at systems in $1+1$ and $2+1$ dimensions with Lagrangeans analogous to 2.1 but with a reduced number of fields. Starting with the lower dimensional case we consider a system of two fields governed by the Langevin equation

$$
\left[\left(\partial_{t}^{2}-\nabla^{2}\right)-\mu^{2}+\lambda \sum_{k=1}^{2} \phi_{k}^{2}+\eta_{i} \partial_{t}\right] \phi_{i}=\xi_{i}, \quad i=1,2
$$

where $\phi_{1}$ is decoupled from the heat bath below $T_{c}$ as in the case we have been discussing. We study the defect formation by subjecting the system to a sudden quench in the temperature of the thermal bath, from a high initial $T_{i}$ to a final low $T_{f}$. This approach captures some of the main features of the continuous transition. In particular, the quench reproduces qualitatively the stage of the transition where the fields do not remain in equilibrium around $T_{c}$ due to critical slowing down.

The quench is accompanied by the decoupling of the $\phi_{1}$ field from the bath. We look for signs of defect formation by looking at the zeroes of the decoupled field following the quench. The three graphs in Figure 5 provide evidence for the formation of kinks when the external dissipation is $\eta_{1}=0.05$ and $\eta_{2}=1$. As the temperature is dropped from $\beta_{\text {initial }}=1$ to $\beta_{\text {final }}=20$ at $t_{\text {quench }}=50$, 

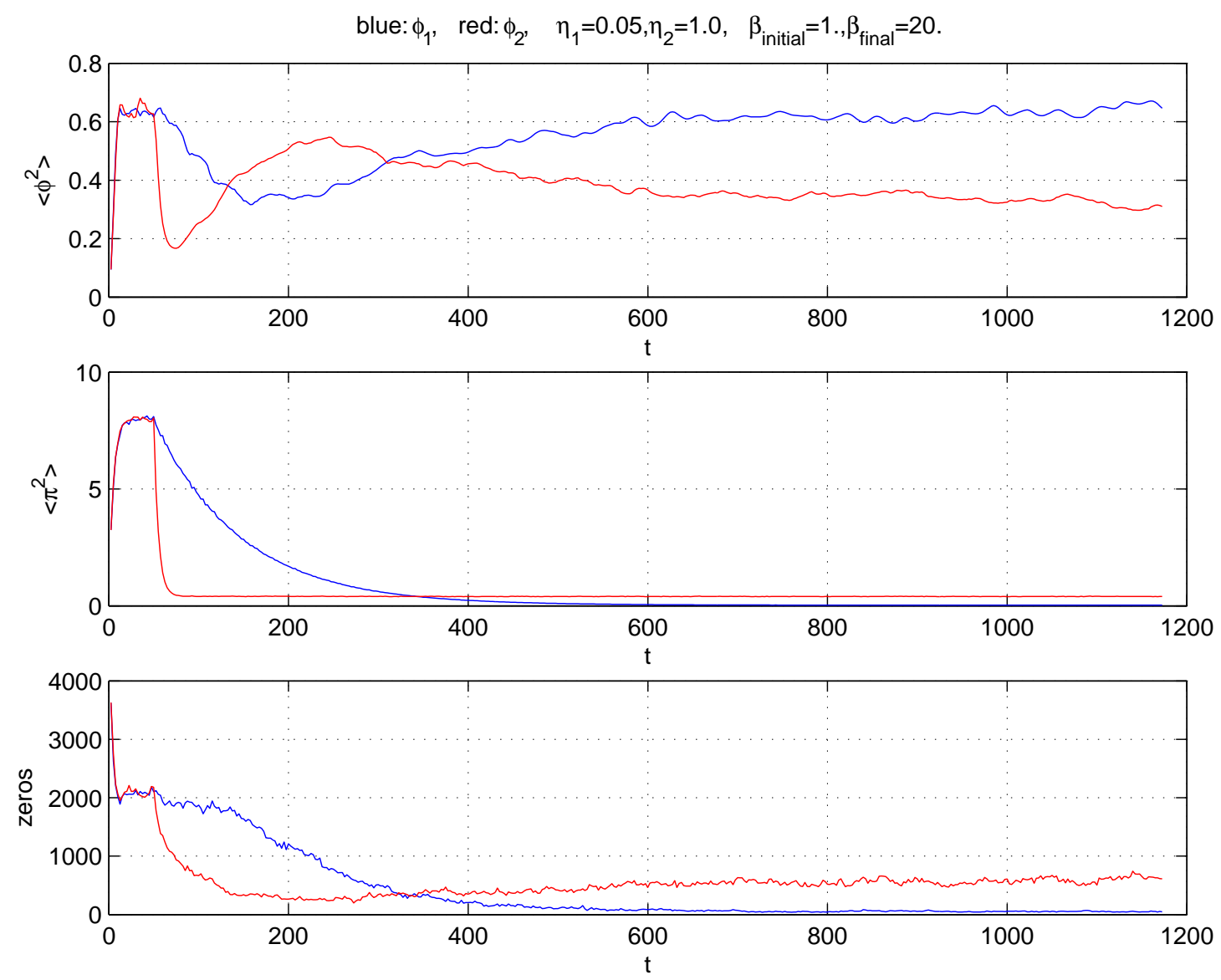

Figure 5

the time evolution before and after the quench is plotted for the square of both fields $\left\langle\phi_{i}^{2}\right\rangle$, for the square of their momentum $\left\langle\pi_{i}^{2}\right\rangle$, and their zeros, respectively. In the first plot we see that $\phi_{1}$ is selected to condense. The second plot shows the decoupled field reaching a steady state with an effective temperature lower than the heat bath temperature. Finally, the third plot shows the number of zero crossings. For the field coupled to the heat bath, this number counting is high because of the thermal fluctuations while for the decoupled one the lower counting corresponds to the actual number of kinks. The presence of the kinks is better illustrated in Figure 6 where we plot the profile of the decoupled field just before the quench and at a later time $t=1200$.

A similar study in $2+1$ dimensions is also been carried out. We consider a three field model where one of the fields $\phi_{1}$ is decoupled from the bath after a quench while the two remaining ones, $\phi_{2}$ and $\phi_{3}$, stay coupled to the bath. In this system we are also observing the formation of defects, now in the form of domain walls. We present results for two values of the external dissipation, $\eta_{1}=1$ and $\eta_{1}=0.1$, and in both cases the direct thermal dissipation is taken to be 1 . We show the time evolutions for $\left\langle\phi_{i}^{2}\right\rangle,\left\langle\pi_{i}^{2}\right\rangle$ and the zero crossings in Figures 7 and 10 for $\eta_{1}=1$ and $\eta_{1}=0.1$ respectively. As in the analogous plots in $1+1$ dimensions shown in Figure 5 there is evidence for defect formation. 

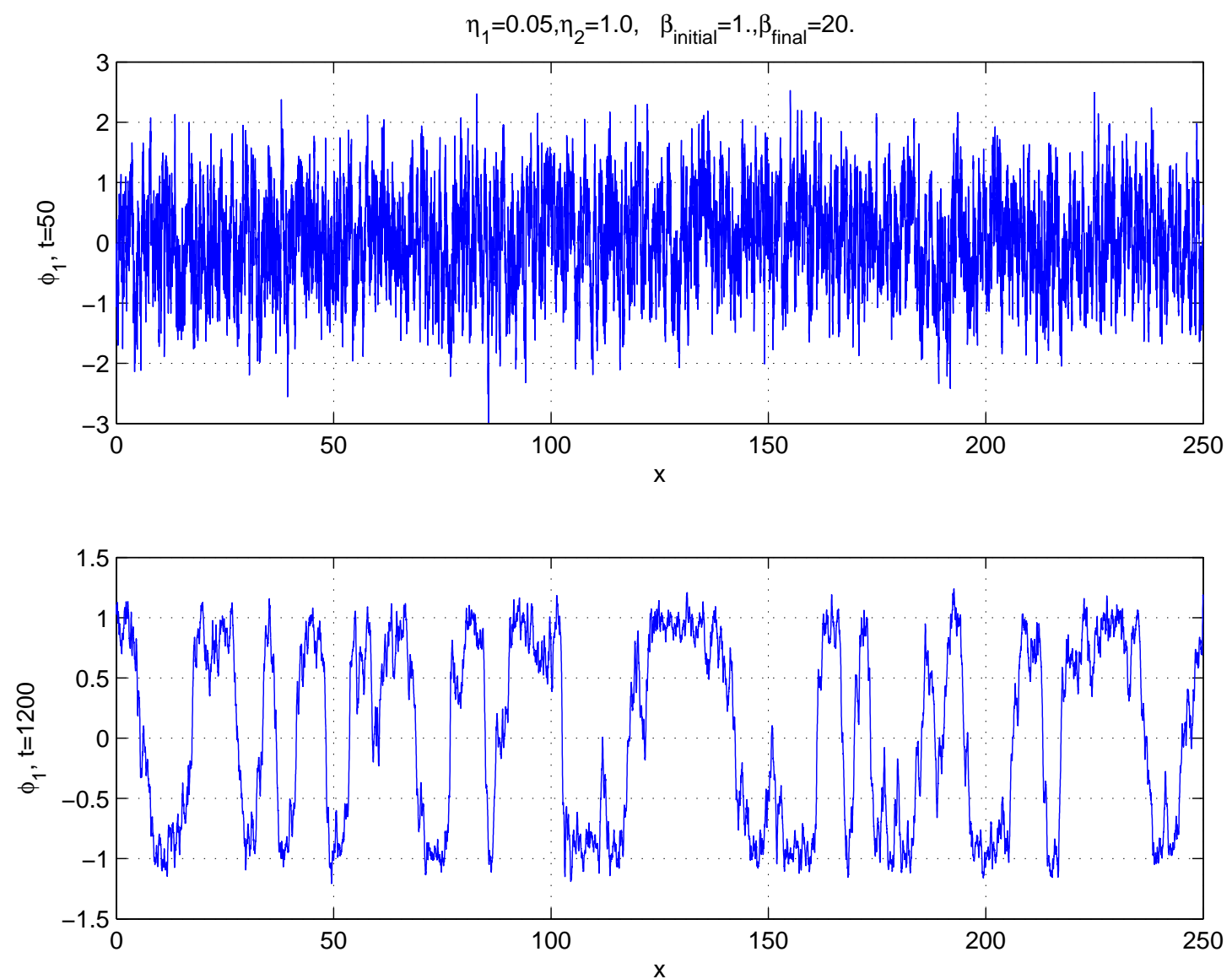

Figure 6

The formation of the domain walls is clearly illustrated in Figures 8 and 9 for the $\eta_{1}=1$. In Figure 8, we show the profile of the decoupled field and in Figure 9 the total potential energy. The zero crossings of the decoupled field overlap with the loci of the maxima of the potential energy as was to be expected. The corresponding plots for $\eta_{1}=0.1$ are shown in Figures 11 and 12. In both cases, the presence of the domain walls is much more tenuous in the figure for the potential due to the large thermal fluctuations of the fields coupled to the bath which almost wash out the defects. The effect increases as the external dissipation is reduced.

The profiles for a lower value of external dissipation also show a decrease in the density of domain walls. In order to improve the visualisation of profiles for even lower values of $\eta_{1}$ we would require using larger lattices. One last effect that our simulations are revealing is an increase in the rate of annihilation of domain walls as $\eta_{1}$ is lowered. With less dissipation the defects move faster and evaporate quickly. This might indicate a possible limitation for the stabilisation mechanism that we are studying. Further investigation is required to clarify this point. 

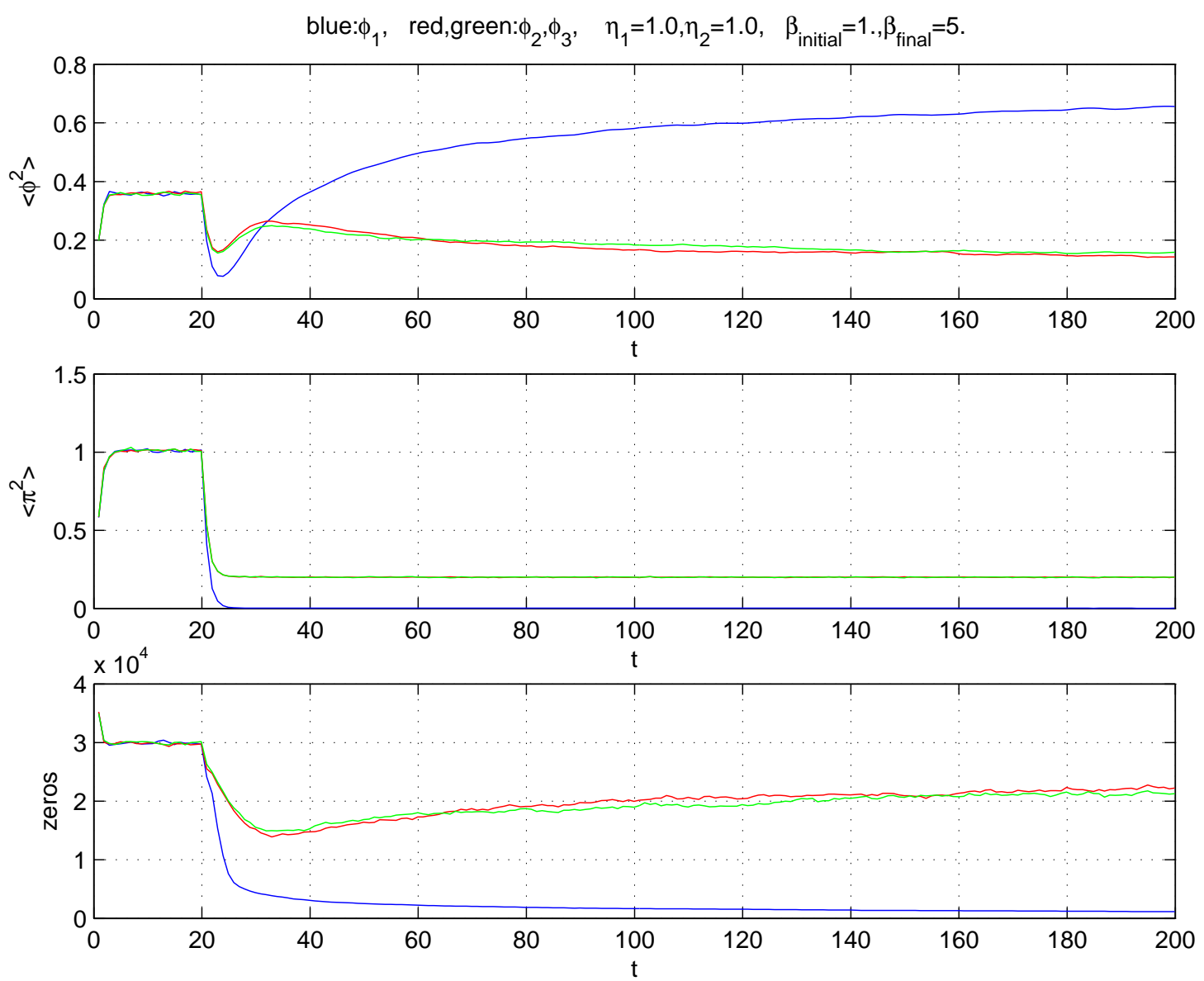

Figure 7

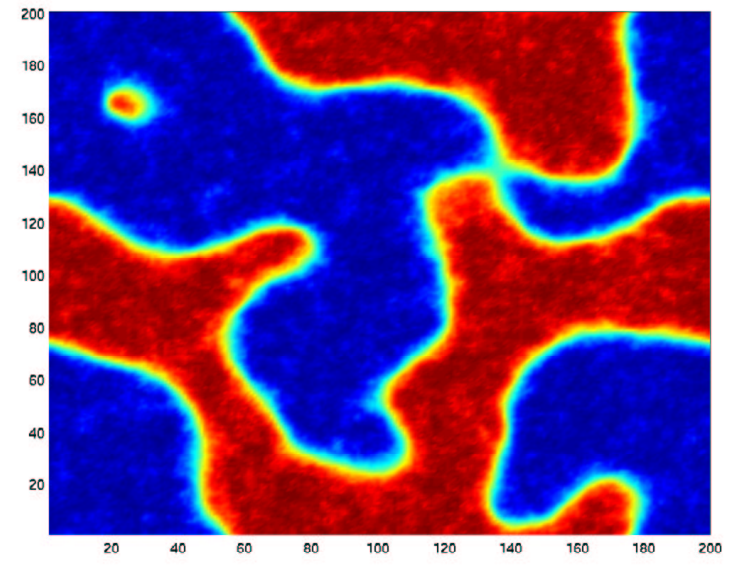

Figure 8

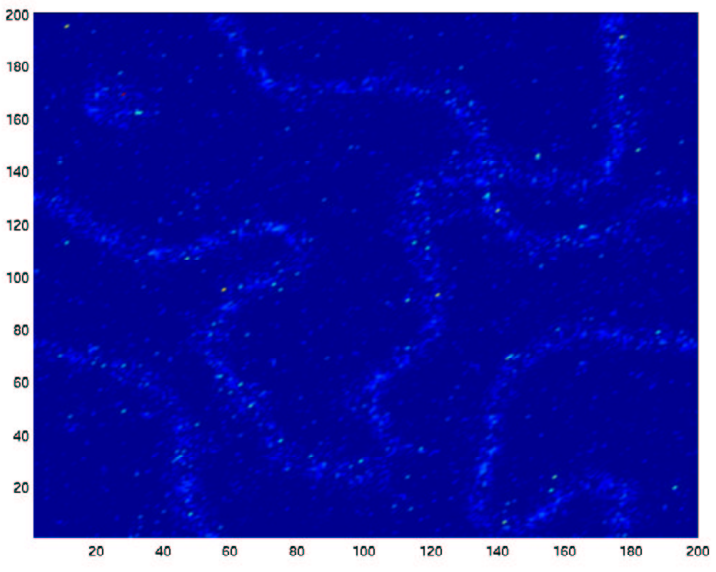

Figure 9 

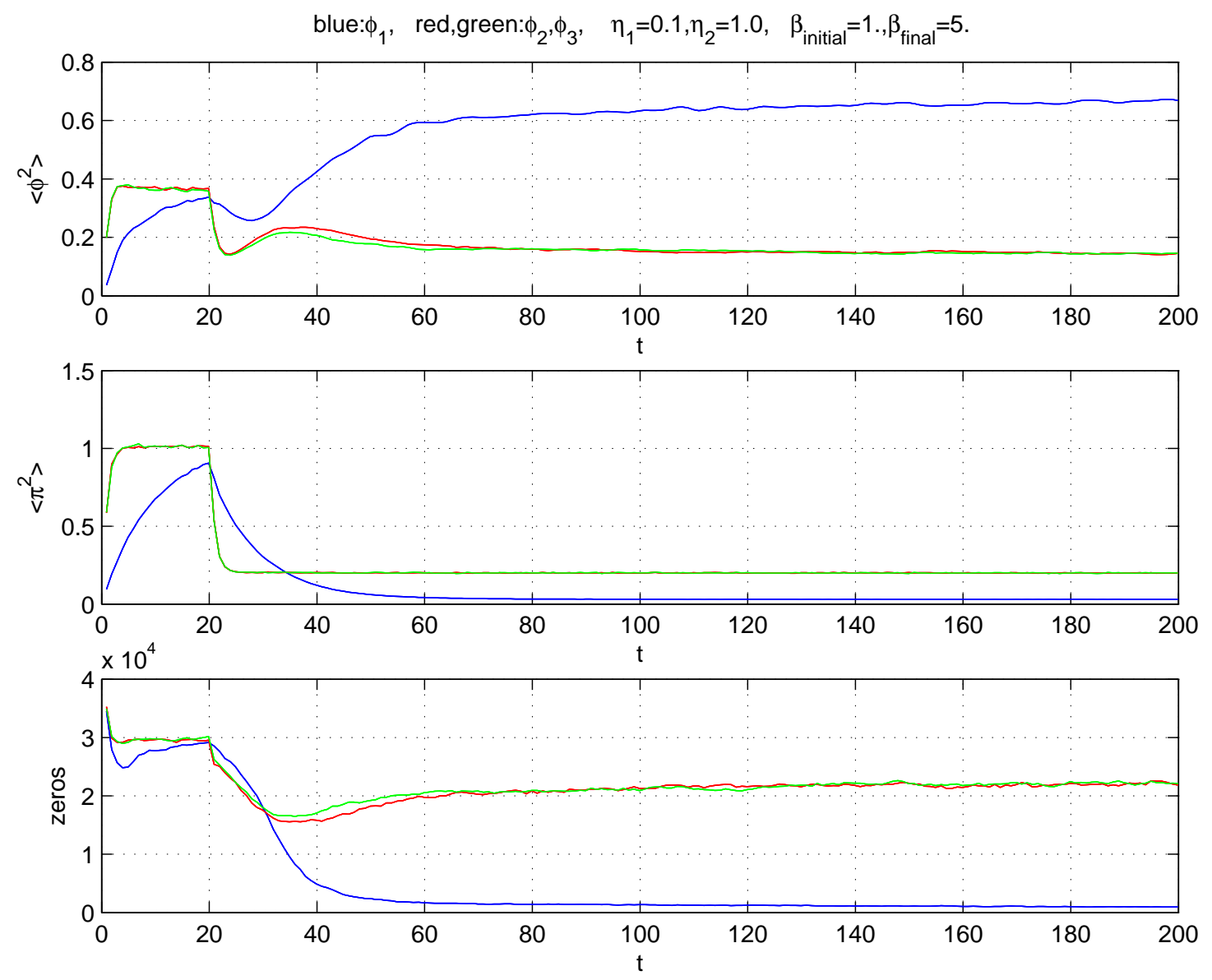

Figure 10

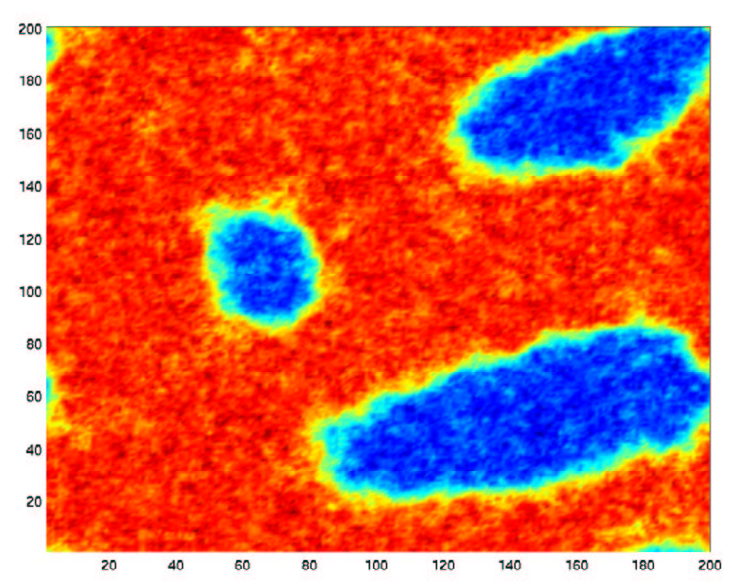

Figure 11

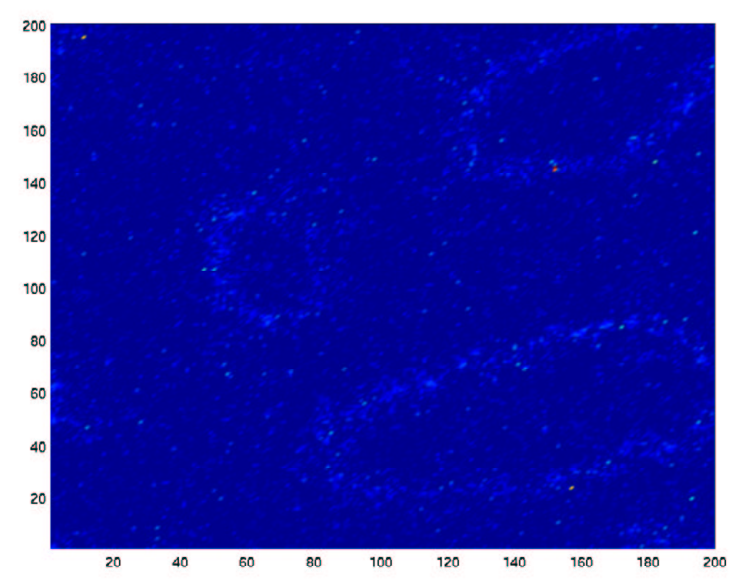

Figure 12 


\section{References}

[1] M. Nagasawa and R. H. Brandenberger, Phys. Lett. B 467 (1999) 205 [hep-ph/9904261]; Phys. Rev. D 67 (2003) 043504 [hep-ph/0207246].

[2] F. Freire, N. D. Antunes, P. Salmi and A. Achúcarro, Phys. Rev. D 72 (2005) 045017 [hep-ph/0502220].

[3] G. N. Felder, J. Garcia-Bellido, P. B. Greene, L. Kofman, A. D. Linde and I. Tkachev, Phys. Rev. Lett. 87 (2001) 011601 [hep-ph/0012142].

[4] D. Boyanovsky and H. J. de Vega, Phys. Rev. D 47 (1993) 2343 [hep-th/9211044]; D. Boyanovsky, D. S. Lee and A. Singh, Phys. Rev. D 48 (1993) 800 [hep-th/9212083]; D. Boyanovsky, H. J. de Vega and R. Holman, Topological defects and the non-equilibrium dynamics of symmetry breaking phase transitions, Les Houches 1999, 139-169 [hep-ph/9903534].

[5] M. Gleiser, Phys. Rev. Lett. 73 (1994) 3495 [hep-ph/9403310]; J. Borrill and M. Gleiser, Phys. Rev. D 51 (1995) 4111 [hep-ph/9410235].

[6] N. D. Antunes, L. M. A. Bettencourt and M. Hindmarsh, Phys. Rev. Lett. 80 (1998) 908 [hep-ph/9708215].

[7] R. J. Rivers and F. C. Lombardo, "How phase transitions induce classical behaviour", hep-th/0412259.

[8] R. Holman, S. Hsu, T. Vachaspati and R. Watkins, Phys. Rev. D 46 (1992) 5352 [hep-ph/9208245].

[9] M. Gleiser and R. O. Ramos, Phys. Rev. D 50 (1994) 2441 [hep-ph/9311278]. A. Berera, M. Gleiser and R. O. Ramos, Phys. Rev. D 58 (1998) 123508 [hep-ph/9803394].

[10] C. Greiner and B. Muller, Phys. Rev. D 55 (1997) 1026 [hep-th/9605048]. 